\title{
Transtibial prosthetic socket design and suspension-mechanism: A literature review
}

\author{
Al Shuaili N (CPO); McGarry A (PhD); Aslani N; Duff L (CPO) \\ Department of Biomedical Engineering, University of Strathclyde Wolfson Centre, \\ Glasgow, United Kingdom.
}

\section{Abstract:}

Study design: A literature review.

Background: The body-weight of the prosthetic user is supported and distributed by the prosthetic socket during the stance phase of gait. Throughout swing phase, inertial forces (pressure and shear) are exerted by the socket suspension-mechanism onto the residuum to facilitate suspension.

Objectives: To identify and investigate available evidence in Trans-Tibial (TT) socket design and suspension to highlight the most effective weight transfer mechanisms and suspension techniques.

Methods: A literature research was conducted comprising two parts: socket design and suspension. Boolean search terms and truncation were used using relevant keywords in online search engines to obtain precise results.

Results: 17 papers which met inclusion criteria were reviewed.

Conclusions: A conclusion on whether socket preference is due to the suspensionmechanism or socket design itself cannot be drawn. PTB sockets are still successfully used and in some studies preferred over TSB. Biomechanically, however, TSB sockets allow for a more even weight-distribution when combined with suction, particularly VASS. Some limited evidence exists to support that such designs may have some effect on wound healing and early ambulation. Further research must be conducted to standardise acclimation periods. Crossover randomised controlled trials (RCT) with larger sample sizes are required to establish an evidence base to improve clinical practice. 
Abstract word count: 207 words.

Abbreviations: Trans-Tibial (TT), Patellar Tendon Bearing (PTB), Total Surface Bearing (TSB), Computer-Aided-Design and Computer-Aided-Manufacture (CAD/CAM), Supracondylar Suspension Patellar Tendon Bearing (SCPTB) Suprapatellar Supracondylar Patellar Tendon Bearing (SC/SP/PTB), (Pressure (P) =Force (F) /Area (A)), Silicone-Liner (SL)/Suction Suspension System (SSS/3S), Hypobaric Iceross Suction (HIS), Vacuum Assisted Suction Suspension (VASS), Icelandic Roll On Silicone Sockets (ICEROSS) Energy Cost of Walking (ECW), Trans-Femoral (TF), Patient Evaluation Questionnaire (PEQ)

\section{Introduction:}

Lower limb amputation is a challenging consequence of diabetes and dysvascular disease ${ }^{1}$. The incidence of TT amputation has reduced in the UK, however it remains the most common amputation level, according to the latest statistics ${ }^{1}$. Following amputation, function and cosmesis are replaced by an unnatural biomechanical device; the prosthesis $^{2,3}$. TT prostheses consist of four main parts: the prosthetic socket and its interface, suspension-mechanism, pylon and foot ${ }^{4}$.

In bipedal gait, body-weight is loaded axially through the musculoskeletal system. In a person with TT amputation, transmission of weight is facilitated through the residual limb soft tissue to the skeleton via the prosthetic socket's interface ${ }^{5}$.

During stance phase, user's body-weight is supported and comfortably distributed within the prosthetic socket by means of its design. By contrast, throughout swing phase, inertial forces (pressure and shear) exerted by the socket and its suspension-mechanism onto the residuum suspend the prosthesis ${ }^{6,7}$. Intolerable lengths of exposure to high levels of stress may cause skin irritation or breakdown. This may be further exacerbated by persistent perspiration that is associated with the use of $\operatorname{SLs}^{8,9,10}$. 


\section{Historical background:}

Two overarching design concepts are known in TT socket design. The PTB socket principles were introduced ${ }^{3}$. This design was the first to facilitate 'total contact' of the prosthetic socket by enclosing the residual limb as a whole. Total contact reduces the risk of oedema and skin problems primarily by the 'pumping effect' the socket creates during ambulation. This aids venous return, reducing the risk of oedema whilst increasing the proprioceptive feedback and hence control of the prosthesis ${ }^{5,69}$.

Although PTB socket is in total contact with the residuum, pressure throughout the socket is not evenly distributed. Weight is borne over anatomically pressure tolerant areas and offloaded from pressure intolerant/sensitive areas ${ }^{6,9}$. Residuum shape can be captured by a variety of methods including: a hands-on casting using plaster of Paris bandages as the prosthetist wraps around the residuum and applies pressure as required ${ }^{11}$. Another 'handsoff' method (CAD/CAM) is detailed in Topper's paper ${ }^{12}$.

Traditionally, the prosthesis was suspended by either leather cuff straps or a thigh corset. Whilst these are still used, there have been several developments to the PTB socket. SCPTB completely incorporates the femoral condyles to suspend the prosthesis by virtue of their anatomical shape. SCPTB aims to increase the contact area, especially for shorter residuums, to reduce pressure and provide mediolateral stability in cases of ligament laxity $3,6,13,14$. Another variant is the $\mathrm{SC} / \mathrm{SP} / \mathrm{PTB}$ that extends proximally over the patella, to aid with recurvatum control $^{6,13}$.

The second socket design was introduced approximately three decades later as the TSB suction socket. As total contact sockets distribute weight transfer over the entire residual limb, this is considered to lower peak pressures due to the larger area $(\mathrm{P}=\mathrm{F} / \mathrm{A})^{5,6}$. It is reported that weight is more evenly distributed and borne over the whole residuum to be transmitted through a larger area $^{3}$. Moreover, pressure within the socket is reduced proximally and increased distally to allow for distribution of forces over a greater area, reducing pressure ${ }^{5,6}$. The development of ICEROSS is an example of a TSB socket 
developed by Kristinsson ${ }^{15}$. Residuum's shape is captured by a hands-off casting technique via a pneumatic bladder which distributes pressure evenly onto the residuum ${ }^{11}$.

There are various suction systems utilised in suspending TSB sockets including (SL)/ (SSS/3S) or sleeve suspension ${ }^{13,14}$. Both systems aim for air elimination from the socket through a one-way valve that is dependent on a seal created via the liner and the socket or alternatively via contact from a silicone or gel sleeve on the thigh makes contact with the skin on the thigh to ensure a good seal of negative pressure with a one-way valve (passivesuction) by which air is not allowed inside the system ${ }^{6,13,14}$. Its mechanism is reliant on friction and negative pressure. Other suction types are the HIS suspension and VASS (active suction). With these systems, due to the enclosed environment from the liner, good hygiene is required to prevent skin irritation from sweat build-up and bacterial formation ${ }^{13,16}$.

Casper introduced VASS technology in the late 1990s. Suction Suspension is enhanced by a vacuum that actively elevates negative pressure and simultaneously controls forces by a mechanical or electrical pump to actively evacuate air from the socket ${ }^{17}$.

Finally, HIS features a hypobaric seal attached to the liner that obviates the need for a sleeve to achieve suction, hence, improves knee flexion RoM and user satisfaction ${ }^{18,19}$.

Locking liners (pin-lock) have been used successfully with both PTB/TSB sockets whereby a pin is attached to the distal end of the liner that connects to a shuttle-lock incorporated within the prosthesis ${ }^{13}$. The 'milking phenomenon' is associated with pinlocking liners and is identified as a downward pull that is exerted on the residual skin by the umbrella-shaped distal end of the liner, at which the locking pin is attached. This creates peak pressures during swing phase and when seated, resulting in discomfort, pain and potentially skin problems like blistering due to continuous cyclic shear ${ }^{20,21,22,23}$.

In 1965 Foort stated that up to $90 \%$ of persons with TT amputation may benefit from a PTB socket ${ }^{3,24}$. Additionally, Osman et al., (2010) concluded that different depths of Patellar tendon (PT) bar had an insignificant effect on the overall distribution of pressure within the socket abs therefore speculated it will be eliminated in the future ${ }^{25}$. Overall, 
PTB socket remains the most frequent prescription ${ }^{3}$. Furthermore, Kristinsson ${ }^{15}$ stated that numerous suspension-mechanisms have been developed to reduce risk of suspension inadequacy within PTB sockets, yet, none of these were considered effective prior to the introduction of suction suspension ${ }^{15}$. However, Hall et al., (2008) reported that roughly 91\% of all TT prosthetic users of TSB socket with SLs experienced a dermatological problem at least once on their residuum ${ }^{26}$.

\section{Biomechanics:}

Optimum effectiveness of PTB socket cannot be achieved by sole loading of the PT bar. As most TT residuum's tolerate minimal, if any, distal end bearing, additional pressure tolerant areas must all be loaded ${ }^{6}$. These are the medial tibial flare, fibular shaft, residual pretibial muscles, and popliteal area ${ }^{3,5,6}$.

In PTB socket, application of a vertical support force to the PT bar, according to Newton's first law of motion; results in a downward and backward motion of the residual limb 5 . Therefore, a counteracting anteriorly directed force is applied to the popliteal area by incorporating an adequately high, flattened posterior wall, with an inward bulge to sufficiently compress soft tissue and eliminate motion ${ }^{5,6}$. Pressure in TSB sockets is more equally distributed throughout the surface of the residuum. Increased pressure on tissues surrounding the weight tolerant areas is believed to reduce pressure on intolerant areas. Weight distribution in TSB sockets is significantly reliant on the choice of interface ${ }^{6}$.

Hydrostatic shape capture techniques originate from the TSB socket principle introduced by Kristinsson and developed by Klasson ${ }^{2,14}$. Based on Pascal's Principle, that states: external pressures are uniformly transmitted through a confined fluid in all directions perpendicularly to the surface of the container ${ }^{4,5}$. Theoretically, the concept of hydrostatics presumes that the residual limb's soft tissue behaves as fluid and abides by the fluid principle, while the hydrostatic system is replicated by the socket. Therefore, when the system is loaded, pressure is distributed equally and peak pressure areas are eliminated to enhance comfort ${ }^{3,15,27}$. 
$\operatorname{Murdoch}^{28}$ (1965), was the first to employ the hands-off principle when the Dundee socket was introduced. In an aim to eliminate positive cast modification and reduce manufacturing time, a fluid-filled tank in which the amputee placed their residuum in weight-bearing conditions ${ }^{28}$ was utilised and the PT bar was incorporated resulting in a TSB socket. Klasson developed this approach by abiding Pascal's law of fluid dynamics resulting in a TSB socket without the PT bar. Another Hands-off shape capture technique ${ }^{29}$, a pneumatic bladder that encapsulates the residual limb with the subject seated. Hands-off techniques are believed to encourage consistency as Hands-on techniques heavily rely on the prosthetist's dexterity, knowledge and skill ${ }^{29}$.

A literature review was conducted to investigate areas of discrepancy in the present knowledge; with regards to available evidence in both TT socket design and suspensionmechanisms to identify the most effective weight transfer mechanism and suspension techniques where possible. The paper will report the most effective weight transfer mechanism as reported in comfort by both users and the prosthetist and minimised pistoning to account for the most effective suspension. Nevertheless, all elements in the prosthesis are equally crucial for optimum function, prosthetic safety and satisfaction, including: choice of suitable socket design, suspension-mechanism, prosthetic foot, alignment and cosmesis ${ }^{6}$.

\section{Methodology:}

The review comprises of two parts: socket design and suspension. It is subdivided to: previous literature reviews, history, rationale and advantages and disadvantages of each. Boolean's searching and truncation were employed to main keywords (diagram 1) in online search engines to obtain precise results. 


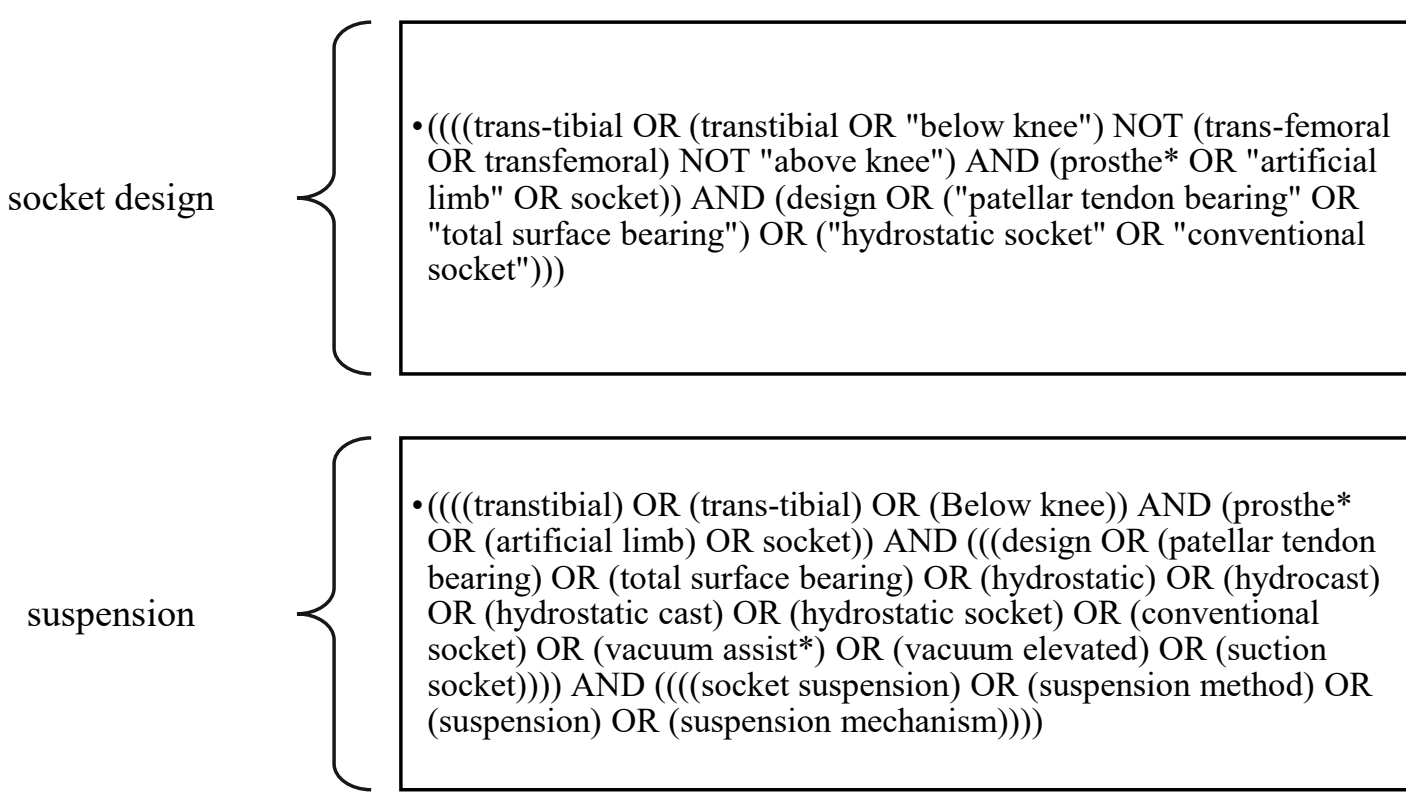

Diagram 1: main keywords

\section{$\underline{\text { Review strategy: }}$}

In each search engine, the search strategy was refined. A total of 135 papers were retrieved relating to inclusion/exclusion criteria (Table 1). Further refined by checking title and abstract. Pubsage/Sciencedirect were used to retrieve literature from chosen studies. Included papers were appraised and graded using the Scottish Intercollegiate Guidelines Network (SIGN) checklists (https://www.sign.ac.uk). Details are provided in the review flowchart (diagram 2). 
Table 1. Inclusion exclusion criteria.

\begin{tabular}{|l|l|}
\hline Inclusion criteria & Exclusion criteria \\
\hline -Everything relevant or match to aim of research. & . Shape capture and consistency. \\
-Socket designs (PTB, TSB) & -TT prosthetics in developed countries. \\
-Biomechanics of socket design. & -Articles that specifics on interface pressure \\
-History of design development, advantages and & measurement, alignment, and gait outcome measures. \\
disadvantages of each design & \\
- Suspension-mechanisms and their advantages and \\
disadvantages
\end{tabular}




\section{Review flowchart:}

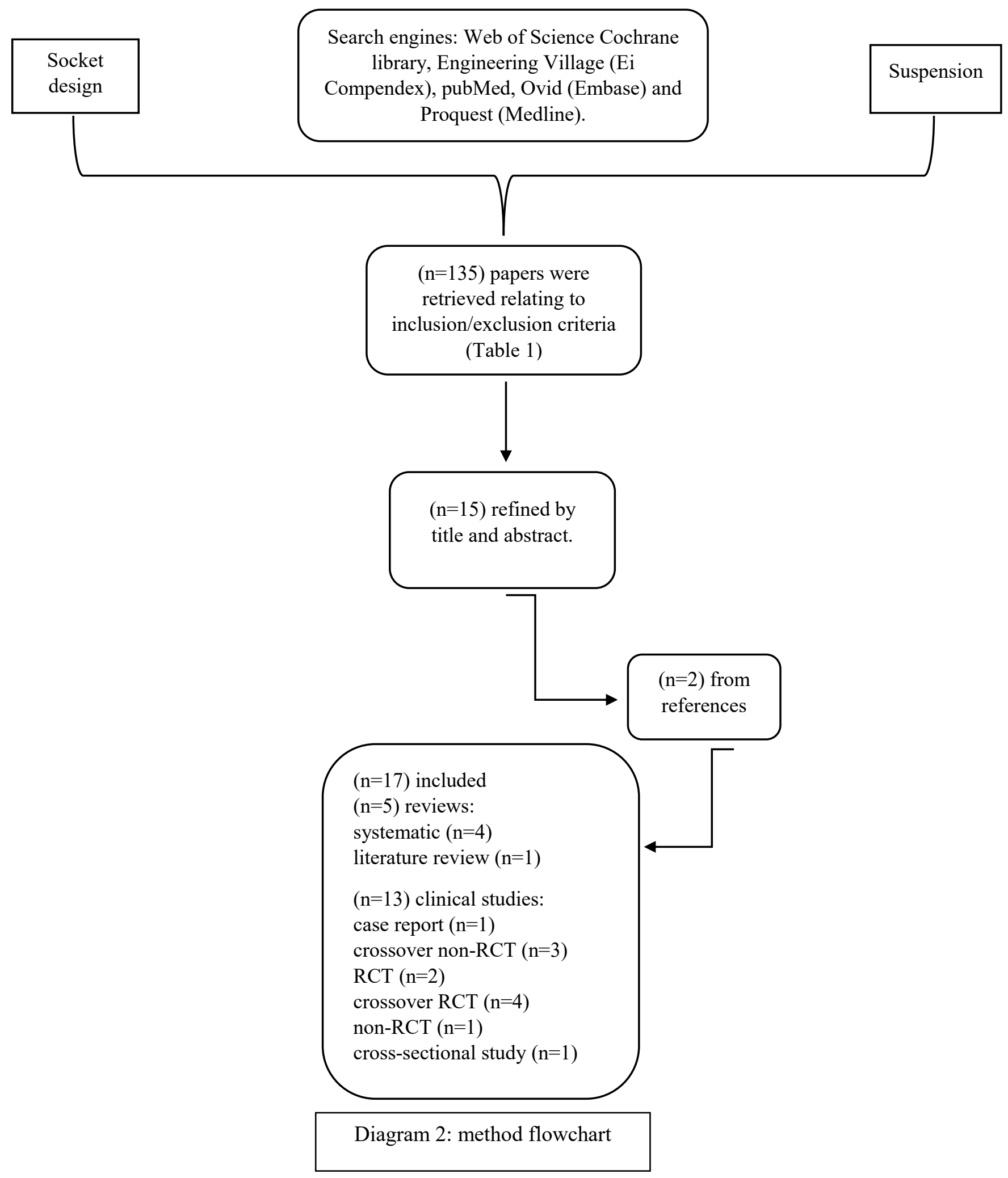




\section{$\underline{\text { Results: evidence tables }}$}

Table 2: literature reviews on socket design

\begin{tabular}{|c|c|c|c|c|c|c|c|c|c|}
\hline $\begin{array}{l}\text { Bibliographic } \\
\text { citation/ } \\
\text { Methodology }\end{array}$ & $\begin{array}{l}\text { SIGN } \\
\text { grade }\end{array}$ & Aim & $\begin{array}{l}\text { Population studied } \\
1 \text { - number }=n \\
\text { 2- average age } \\
\text { 3- sex male : female } \\
\text { 4- cause of amputation } \\
\text { (respectively) }\end{array}$ & $\begin{array}{l}\text { Intervention } \\
\text { socket design and } \\
\text { number of studies / } \\
\text { suspension and } \\
\text { number of studies } \\
\text { Duration of study } \\
\end{array}$ & Outcomes & $\begin{array}{l}\text { Limitations/ } \\
\text { errors }\end{array}$ & $\begin{array}{l}\text { Search method } \\
\text { 1-databases used } \\
\text { 2-included } \\
\text { articles }\end{array}$ & $\begin{array}{l}\text { Tool used to } \\
\text { assess } \\
\text { quality of } \\
\text { literature, if } \\
\text { any. }\end{array}$ & $\begin{array}{l}\text { Were all } \\
\text { outcomes } \\
\text { measured }\end{array}$ \\
\hline $\begin{array}{l}\text { (Safari and } \\
\text { Meier, 2015) } \\
\text { (part 1) } \\
\text { Systematic } \\
\text { approach in } \\
\text { appraising } \\
\text { and sourcing } \\
\text { literature }\end{array}$ & \multirow[t]{2}{*}{$1+$} & \multirow[t]{2}{*}{$\begin{array}{l}\text { Simplify } \\
\text { socket fit } \\
\text { and } \\
\text { determine } \\
\text { chief } \\
\text { characteristic } \\
\text { of a } \\
\text { successful } \\
\text { fitting and } \\
\text { provide an } \\
\text { indication } \\
\text { for } \\
\text { prescription }\end{array}$} & $\begin{array}{l}1-\mathrm{n}=790 \\
2-50.7 \text { years old } \\
3-592: 151 \\
\text { 4-Trauma, vascular } \\
\text { insufficiency, diabetes others. }\end{array}$ & $\begin{array}{l}- \text { TSB }(n=8) \\
\text {-PTB vs TSB }(n=5) \\
\text {-TSB/VAS }(n=3) \\
\text {-TSB/HS vs either } \\
\text { PTB or TSB }(n=3) \\
\\
\text { Duration: } \\
(n=3) \text { not reported } \\
(n=7) \leq 3 \text { mo } \\
(n=4) \geq 3 \text { mo } \\
(n=5) \text { inconsistent }\end{array}$ & \multirow[t]{2}{*}{$\begin{array}{l}\text { (flowchart } \\
\text { available in } \\
\text { part 1) }\end{array}$} & \multirow[t]{2}{*}{$\begin{array}{l}\text { large } \\
\text { heterogeneity in } \\
\text { papers in } \\
\text { design, } \\
\text { population, } \\
\text { outcome etc } \\
\text { therefore no } \\
\text { meta-analyses } \\
\text { was conducted }\end{array}$} & \multirow{2}{*}{$\begin{array}{l}\text { Useful flow } \\
\text { diagram in part } \\
1 \text { of the study } \\
\text { (1998- July } \\
\text { 2013) } \\
\text { Databases used } \\
\text { ( } n=5 \text { ): Medline } \\
\text { (PubMed), } \\
\text { Embase (Ovid } \\
\text { Interface), } \\
\text { Google Scholar, } \\
\text { the Cochrane } \\
\text { Library and } \\
\text { Web of } \\
\text { Knowledge } \\
\text { (WoK). } \\
\text { Assessed for } \\
\text { eligibility } \\
(n=87) \\
\text { Excluded full } \\
\text { text ( } n=53) \\
\text { Included } \\
(n=35):-19 \\
\text { qualitative } \\
-27 \text { quantitative } \\
\text { Studies } \\
\text { imported from } \\
\text { reference list of } \\
\text { included study } \\
(n=1)\end{array}$} & \multirow[t]{2}{*}{$\begin{array}{l}\text { Downs and } \\
\text { Black risk of } \\
\text { bias } \\
\text { assessment } \\
\text { checklist }\end{array}$} & \multirow[t]{2}{*}{ Yes } \\
\hline $\begin{array}{l}\text { (Safari and } \\
\text { Meier, 2015) } \\
\text { (part 2) } \\
\text { Systematic } \\
\text { approach in } \\
\text { appraising } \\
\text { and sourcing } \\
\text { literature }\end{array}$ & & & $\begin{array}{l}1-\mathrm{n}=302 \\
2-42.64 \text { years old } \\
\text { 3-162:37 } \\
\text { 4-Trauma, vascular } \\
\text { insufficiency or diabetes, } \\
\text { others. }\end{array}$ & $\begin{array}{l}-\mathrm{PTB}(\mathrm{n}=3) \\
\text {-TSB (n=12) } \\
\text {-TSB/VASS vs TSB } \\
\text {-TSB/HS vs PTB }(\mathrm{n}=2) \\
\text {-TSB/ICEX and TSB } \\
(\mathrm{n}=1) \\
\\
\text { Duration } \\
(\mathrm{n}=6)<3 \text { mo } \\
(\mathrm{n}=6) \geq 3 \text { mo } \\
(\mathrm{n}=5) \text { not considered } \\
(\mathrm{n}=10) \text { not reported }\end{array}$ & & & & & \\
\hline
\end{tabular}


Table 3: clinical studies on socket design

\begin{tabular}{|c|c|c|c|c|c|c|c|c|c|}
\hline $\begin{array}{l}\text { Bibliographi } \\
\text { c citation }\end{array}$ & $\begin{array}{l}\text { SIGN } \\
\text { grade }\end{array}$ & $\begin{array}{l}\text { Study } \\
\text { design }\end{array}$ & Aim & $\begin{array}{l}\text { Participants } \\
\text { characteristics } \\
\text { 1-inclusion criteria } \\
\text { 2-number of } \\
\text { participants } \\
\text { recruited/analysed } \\
\text { 3-Mean age } \\
\text { 4-Male:Female } \\
\text { 5-Cause of amputation } \\
\text { 6-Years using } \\
\text { prosthesis }\end{array}$ & $\begin{array}{l}\text {-Methods } \\
\text {-Acclimation }\end{array}$ & Comparator & $\begin{array}{l}\text { Intervention: } \\
\text { socket design }\end{array}$ & $\begin{array}{l}\text { Outcomes: } \\
\text {-main findings } \\
\text {-limitations }\end{array}$ & $\begin{array}{l}\text { Commercial } \\
\text { bias? }\end{array}$ \\
\hline $\begin{array}{l}\text { Goh et al } \\
(2004)\end{array}$ & $1-$ & $\begin{array}{l}\text { Crossover } \\
\text { non-RCT }\end{array}$ & $\begin{array}{l}\text { Compare } \\
\text { pressure of } \\
\text { PTB vs } \\
\text { HC/TSB } \\
\text { sockets }\end{array}$ & $\begin{array}{l}\text { 1- unilateral TT } \\
\text { 2- }(\mathrm{n}=4) \\
3-N / A \\
4-100 \% \text { male } \\
5 \text { - vascular }(n=1) \\
\text { trauma }(n=3) \\
\text { 6-at least } 5 \text { year } \\
\text { amputation before study }\end{array}$ & $\begin{array}{l}\text { PTB socket } \\
\text { (prosthetist) } \\
\text { HC/TSB } \\
\text { (technician) } \\
\mathbf{1 6} \text { pressure } \\
\text { measurement } \\
\text { sites (transducers) } \\
\text { Tests divided: } \\
\text { static/dynamic } \\
\text { Pressure measure: } \\
\text { static conditions } \\
\text { Three minimum } \\
\text { trials recorded } \\
\text {-same day testing }\end{array}$ & $\begin{array}{l}\text {-Pressure } \\
\text { transducers to } \\
\text { measure socket- } \\
\text { sock interface } \\
\text { stresses } \\
\text {-Gait analysis: } \\
\text { pressure transducers } \\
\text { connected to } \\
\text { VICON motion } \\
\text { system with two } \\
\text { Kistler force } \\
\text { platforms to analyse } \\
\text { gait using infrared } \\
\text { cameras. }\end{array}$ & $\begin{array}{l}\text { PTB vs } \\
\text { TSB/Hydroc } \\
\text { ast. }\end{array}$ & $\begin{array}{l}\text { Subjects individually } \\
\text { analysed. } \\
\text { Figures available to } \\
\text { illustrate pressure } \\
\text { profiles in all planes } \\
\text { and walking } \\
\text { conditions }\end{array}$ & No \\
\hline
\end{tabular}




\begin{tabular}{|c|c|c|c|c|c|c|c|c|c|}
\hline $\begin{array}{l}\text { Selles et al } \\
(2005)\end{array}$ & 1- & $\begin{array}{l}\text { Prospective } \\
\text { RCT }\end{array}$ & $\begin{array}{l}\text { Compare } \\
\text { functional } \\
\text { outcome and } \\
\text { cost } \\
\text { efficiency of } \\
\text { TSB with } \\
\text { PTB }\end{array}$ & $\begin{array}{l}\text { 1- Unilateral TT, over a } \\
\text { year use of prosthesis, } \\
\text { active walkers with or } \\
\text { without walking aid, no } \\
\text { residuum problems, } \\
\text { able to bear pressure on } \\
\text { distal end, had no } \\
\text { reported issues with } \\
\text { silicone liners. } \\
\text { 2- }(\mathrm{n}=36) / \text { analysed } \\
(\mathrm{n}=26) \\
\text { 3- } 60 \mathrm{yr} \\
\text { 4- } \mathrm{n} / \mathrm{a} \\
\text { 5- } 50 \% \text { trauma } \\
\text { 6- } \mathrm{n} / \mathrm{a}\end{array}$ & $\begin{array}{l}\text { Randomly } \\
\text { assigned into: } \\
\text { TSB group and } \\
\text { PTB group } \\
\text { Baseline: Liner } \\
\text { not supplied to all } \\
\text { participants } \\
\text { Randomisation of } \\
\text { this aspect } \\
\text { stratified authors } \\
\text { expected liners } \\
\text { would improve } \\
\text { outcome } \\
-3 \text { months }\end{array}$ & $\begin{array}{l}\text {-PEQ score } \\
\text {-Mobility-related } \\
\text { activities of ADL } \\
\text {-Gait characteristics } \\
\text { (baseline/ 3months) } \\
\text {-Cost of materials } \\
\text {-Manufacturing } \\
\text { time } \\
\text {-Number of } \\
\text { visits/interventions }\end{array}$ & $\begin{array}{l}\text { PTB/pin-lock } \\
\text { TSB/pin-lock }\end{array}$ & $\begin{array}{l}\text { Patient satisfaction: } \\
\text { PEQ, if new } \\
\text { prosthesis kept } \\
\text { Activity monitoring, } \\
\text { gait analysis and } \\
\text { prosthetic mass: } \\
\text { PTB: higher at } \\
\text { baseline } \\
\text { \% hours spent in } \\
\text { dynamic activities } \\
\text { (7.4\%) within } \\
\text { amputees/able-bodied } \\
\text { activity level } \\
\text { Average walking } \\
\text { speed: baseline TSB } \\
\text { slower than average } \\
\text { Large variability in } \\
\text { physical ability, } \\
\text { activity monitoring } \\
\text { results alike } \\
\text { Economic variables: } \\
\text { Production/ Clinician } \\
\text { time: TSB reduced } \\
\text { Visits/material cost: } \\
\text { TSB higher } \\
\text { Limitations: } \\
\text {-Small sample } \\
\text {-Absent interface } \\
\text { pressure testing and } \\
\text { oxygen saturation. }\end{array}$ & Yes \\
\hline $\begin{array}{l}\text { Manucharian } \\
\text { (2011) }\end{array}$ & 1- & $\begin{array}{l}\text { Controlled } \\
\text { trial }\end{array}$ & $\begin{array}{l}\text { Compare } \\
\text { outcomes of } \\
\text { (Hands-on } \\
\text { PTB and } \\
\text { hands-of } \\
\text { Hydrocast) } \\
\text { with regards } \\
\text { to comfort } \\
\text { level }\end{array}$ & $\begin{array}{l}\text { established prosthesis } \\
\text { wearer, unilateral TT, } \\
\text { no open wounds } \\
1-(\mathrm{n}=36) \text { divided them } \\
\text { into (nPTB=21) control } \\
\text { group (nHCTSB=15) } \\
\text { experimental } \\
\text { (n=36) } \\
\text { Characteristics } \\
\text { illustrated in table } 1 . \\
\text {-table } 1 \text { illustrates } \\
\text { age/characteristics } \\
-11: 7 \\
\text {-PVD } 83.6 \% \text {, trauma } \\
16.7 \% \\
-7+\text { to }<2 \text { years } \\
\end{array}$ & $\begin{array}{l}\text { Recruited from } \\
\text { existing patients in } \\
\text { Orthopaedic Arts } \\
\text { Laboratory. } \\
\text { Random allocation } \\
\text { to groups, } \\
\text { randomisation not } \\
\text { detailed }\end{array}$ & $\begin{array}{l}\text { SCS instrument, (at } \\
\text { initial fitting, in } 1 \\
\text { month) } \\
\text { 11-point Likert-type } \\
\text { scale used to } \\
\text { increase } \\
\text { appropriateness of } \\
\text { data for parametric } \\
\text { analysis. }\end{array}$ & $\begin{array}{l}\text { PTB vs } \\
\text { HC/TSB } \\
\text { Both with } \\
\text { Pelite liner, } \\
\text { passive } \\
\text { suction (one- } \\
\text { way valve) }\end{array}$ & $\begin{array}{l}\text {-Differences of initial } \\
\text { and final socket } \\
\text { comfort assessed by } \\
\text { paired } t \text { tests } \\
\text { PTB comfort } \\
\text { statistically higher } \\
\text { Limitations: } \\
\text { No randomisation } \\
\text { Unequal sample } \\
\text { group } \\
\text { Long relationships } \\
\text { with experimenter, } \\
\text { might cause bias } \\
\text { Pelite liners with } \\
\text { both, to reduce } \\
\text { variables }\end{array}$ & No \\
\hline
\end{tabular}


Table 4: literature reviews on suspension/liners

\begin{tabular}{|c|c|c|c|c|c|c|c|c|c|}
\hline $\begin{array}{l}\text { Bibliographic } \\
\text { citation/ } \\
\text { Methodology }\end{array}$ & $\begin{array}{l}\text { SIGN } \\
\text { grade }\end{array}$ & Aim & $\begin{array}{l}\text { Population studied } \\
1 \text { - number }=n \\
\text { 2- average age } \\
\text { 3- sex male : female } \\
\text { 4- cause of amputation } \\
\text { (respectively) }\end{array}$ & $\begin{array}{l}\text { Intervention } \\
\text { socket design and } \\
\text { number of studies / } \\
\text { suspension and } \\
\text { number of studies } \\
\text { Duration of study }\end{array}$ & Outcomes & $\begin{array}{l}\text { Limitations/ } \\
\text { errors }\end{array}$ & $\begin{array}{l}\text { Search method } \\
\text { 1-databases } \\
\text { used } \\
\text { 2-included } \\
\text { articles }\end{array}$ & $\begin{array}{l}\text { Tool used to } \\
\text { assess quality } \\
\text { of literature, } \\
\text { if any. }\end{array}$ & $\begin{array}{l}\text { Were all } \\
\text { outcomes } \\
\text { measured? }\end{array}$ \\
\hline $\begin{array}{l}\text { (Gholizadeh } \\
\text { et al., 2014) } \\
\text { /Systematic } \\
\text { approach }\end{array}$ & $2++$ & $\begin{array}{l}\text { Find scientific } \\
\text { evidence } \\
\text { pertaining to } \\
\text { various TT } \\
\text { suspension } \\
\text { systems and to } \\
\text { provide } \\
\text { selection } \\
\text { criteria for } \\
\text { clinicians. }\end{array}$ & $\begin{array}{l}\text {-Information for all aspects } \\
\text { mentioned for individual } \\
\text { papers, no total numbers } \\
\text { provided }\end{array}$ & $\begin{array}{l}\text { No total figures } \\
\text { Provided } \\
\text { Table } 3 \text { illustrates } \\
\text { details of socket } \\
\text { design/suspension } \\
\text { Duration: } \mathrm{n} / \mathrm{a}\end{array}$ & $\begin{array}{l}\text { Total }(\mathrm{n}=516) \\
\text { Title/abstract } \\
\text { assessed }(\mathrm{n}=22) \\
(\mathrm{n}=45) \text { from } \\
\text { reference lists } \\
(\mathrm{n}=9) \text { suitable } \\
\text { Total }(\mathrm{n}=31) \\
\text { included } \\
\text { Surveys ( } \mathrm{n}=7) \\
\text { Clinical studies } \\
(\mathrm{n}=24)\end{array}$ & $\mathrm{N} / \mathrm{a}$ & $\begin{array}{l}\text { Web of Science, } \\
\text { ScienceDirect } \\
\text { and PubMed }\end{array}$ & $\begin{array}{l}\text { Adapted Van } \\
\text { der Linde et } \\
\text { al to be used } \\
\text { for gathered } \\
\text { study designs } \\
(13 \text {-element } \\
\text { checklist }) \\
(\mathrm{n}=9) \text { failed, } \\
\text { rest classified } \\
\text { into either A- } \\
\text { level, B-level } \\
\text { or C-level }\end{array}$ & Yes \\
\hline $\begin{array}{l}\text { (Richardson } \\
\text { and Dillon, } \\
\text { 2017) } \\
\text { Systematic } \\
\text { approach }\end{array}$ & $1+$ & $\begin{array}{l}\text { Critically } \\
\text { appraise and } \\
\text { synthesise } \\
\text { research } \\
\text { describing } \\
\text { user } \\
\text { experience of } \\
\text { TT prosthetic } \\
\text { liners. }\end{array}$ & $\begin{array}{l}\text { Total not provided } \\
\text { Reviewer noted from table } \\
2 \\
1 \text { - }(\mathrm{n}=757) \\
\text { 2-Between } 62-42 \\
\text { 3-Mostly male, illustrated } \\
\text { in \% lowest } 70 \% \\
\text { 4-Illustrated in \%, trauma, } \\
\text { PVD/DM, other, tumour. }\end{array}$ & $\begin{array}{l}\text { No total figures. } \\
\text { Table } 3 \text { provides } \\
\text { summary of studies }\end{array}$ & $\begin{array}{l}\text { PRISMA } \\
\text { Flowchart } \\
\text { available) } \\
\text { identified } \\
(\mathrm{n}=1530) \text {, only } \\
(\mathrm{n}=18) \text { included: } \\
(\mathrm{n}=1) \text { identified } \\
\text { from reference lists }\end{array}$ & $\begin{array}{l}\text { 1-Single } \\
\text { reviewer } \\
\text { completed } \\
\text { the search } \\
\text { and sat } \\
\text { inclusion } \\
\text { criteria. } \\
\text { 2-Narrative } \\
\text { approach } \\
\text { adopted due } \\
\text { to limitations } \\
\text { in existing } \\
\text { evidence, no } \\
\text { statistical } \\
\text { analysis } \\
\text { possible. }\end{array}$ & $\begin{array}{l}\text { MEDLINE } \\
\text { (Ovid), } \\
\text { CINAHL } \\
\text { (EBSCO), } \\
\text { EMBASE } \\
\text { (Ovid), the } \\
\text { Cochrane } \\
\text { Library (Update } \\
\text { Software Ltd), } \\
\text { ProQuest } \\
\text { Nursing and } \\
\text { Allied Health, } \\
\text { AMED (Ovid), } \\
\text { and SCOPUS } \\
\text { (Elsevier). }\end{array}$ & $\begin{array}{l}\text { McMaster } \\
\text { University } \\
\text { Critical } \\
\text { Review Form }\end{array}$ & Yes \\
\hline
\end{tabular}




\begin{tabular}{|c|c|c|c|c|c|c|c|c|c|}
\hline $\begin{array}{l}\text { (Baars and } \\
\text { Geertzen, } \\
2005 \text { ) } \\
\text { Literature } \\
\text { review }\end{array}$ & $2++$ & $\begin{array}{l}\text { Find objective } \\
\text { documentation } \\
\text { in support of } \\
\text { the advantages } \\
\text { in prosthetic } \\
\text { fitting/use of } \\
\text { silicone liners }\end{array}$ & $\begin{array}{l}\text { Total figures not provided, } \\
\text { Reviewer found: } \\
\text { 1-(n=259) } \\
\text { 2-Ranged between (27.8- } \\
\text { 70) } \\
\text { 3-N/a } \\
\text { 4-Illustrated by \%, trauma, } \\
\text { vascular, diabetes, others }\end{array}$ & $\begin{array}{l}\text { Total figures not } \\
\text { provided } \\
\text { Reviewer noted } \\
\text { from table } 4 \text { : } \\
\text { 1-SL liner }(\mathrm{n}=4) \\
\text { SL, Fillauer } \\
\text { silicone suspension } \\
\text { liner } \\
\text { Silicone suction } \\
\text { socket }(\mathrm{n}=1) \\
\text { 2-Liner type } \\
\text { unknown/not } \\
\text { mentioned }(\mathrm{n}=2)\end{array}$ & $\begin{array}{l}\text { Total }(\mathrm{n}=132) \\
(\mathrm{n}=6) \text { remained }\end{array}$ & $\mathrm{N} / \mathrm{a}$ & $\begin{array}{l}\text { Medline, } \\
\text { Embase, Amed, } \\
\text { Cochrane and } \\
\text { Cinahl }\end{array}$ & None & Yes \\
\hline
\end{tabular}


Table 5: clinical studies on suspension

\begin{tabular}{|c|c|c|c|c|c|c|c|c|c|}
\hline $\begin{array}{l}\text { Bibliographi } \\
\text { c citation }\end{array}$ & $\begin{array}{l}\text { SIGN } \\
\text { grade }\end{array}$ & $\begin{array}{l}\text { Study } \\
\text { Design }\end{array}$ & Aim & $\begin{array}{l}\text { Participants } \\
\text { characteristics } \\
\text { 1-inclusion criteria } \\
\text { 2-number of } \\
\text { participants } \\
\text { recruited/analysed } \\
\text { 3-Mean age } \\
\text { 4-Male:Female } \\
\text { 5-residuum } \\
\text { condition } \\
\end{array}$ & $\begin{array}{l}\text {-Method } \\
\text {-Acclimation }\end{array}$ & $\begin{array}{l}\text { Intervention } \\
\text { (suspension- } \\
\text { mechanism) }\end{array}$ & Comparator & $\begin{array}{l}\text { Outcomes } \\
\text {-benefits } \\
\text {-negative outcomes } \\
\text {-limitations }\end{array}$ & $\begin{array}{l}\text { Commercial } \\
\text { bias? }\end{array}$ \\
\hline $\begin{array}{l}\text { Narita et al } \\
\text { (1997) }\end{array}$ & 3 & $\begin{array}{l}\text { Cross- } \\
\text { sectional }\end{array}$ & $\begin{array}{l}\text { Compare } \\
\text { suspension of } \\
\text { PTB/unknown } \\
\text { vs TSB/pin- } \\
\text { lock using x- } \\
\text { ray and } \\
\text { cineradiograp } \\
\text { hy }\end{array}$ & $\begin{array}{l}\text { 1-Used TSB at least } \\
6 \text { months before trial } \\
2-(n=9) \text { one } \\
\text { bilateral=10 } \\
\text { limbs/ }(n=3) \\
3-33.9 \text { years } \\
4-8: 1 \\
5-n / a\end{array}$ & $\begin{array}{l}\text { Lateral view x-rays } \\
\text { taken of prosthesis } \\
\text { with a } 5 \mathrm{~kg} \text { mass at } \\
30^{\circ} \text { of knee flexion. } \\
\text { The distance } \\
\text { between tibial end } \\
\text { and the base of the } \\
\text { socket was then } \\
\text { measured. }\end{array}$ & $\begin{array}{l}\mathrm{N} / \mathrm{A} \\
\text { (observation } \\
\text { al) }\end{array}$ & $\begin{array}{l}\text {-Tibial } \\
\text { displacement: } \\
\text { x-ray } \\
\text {-Suspension } \\
\text { /tibial stability: } \\
\text { cineradiograph } \\
\text { y }\end{array}$ & $\begin{array}{l}\text { X-ray comparison: } \\
\text { TSB significantly less } \\
\text { Cineradiography } \\
\text { comparison: } \\
\text { TSB/pin-lock better } \\
\text { Cineradiography } \\
\text { comparison of tibial } \\
\text { stability within socket: } \\
\text { TSB anteroposterior } \\
\text { stability greater; TSB } \\
\text { statistically smaller angle }\end{array}$ & No \\
\hline $\begin{array}{l}\text { Coleman et } \\
\text { al (2004) }\end{array}$ & $1+$ & $\begin{array}{l}\text { Randomised } \\
\text { crossover }\end{array}$ & $\begin{array}{l}\text { Comparison } \\
\text { between TT } \\
\text { suspension } \\
\text { systems: } \\
\text { TSB/Alpha } \\
\text { liner/pin-lock } \\
\text { versus } \\
\text { PTB/Pe- } \\
\text { lite/passive- } \\
\text { suction }\end{array}$ & $\begin{array}{l}\text { 1-unilateral TT, at } \\
\text { least one year since } \\
\text { amputation, } \\
\text { traumatic Compliant } \\
\text { prosthetic users, no } \\
\text { major health } \\
\text { problems and } \\
\text { minimum activity } \\
\text { level } 2 \text { on (Durable } \\
\text { Medical Equipment } \\
\text { Regional Carrier) } \\
\text { 2- }(\mathrm{n}=14) /(\mathrm{n}=13) \\
\text { 3- } 49.4 \\
\text { 4-13:7 } \\
\text { 5-n/a }\end{array}$ & $\begin{array}{l}\text {-Pairs } \\
\text { simultaneously } \\
\text { assigned into the } \\
\text { protocol randomly to } \\
\text { control seasonal } \\
\text { variability } \\
\text {-Exit interview } \\
\text { conducted after } \\
\text { subjects chose } \\
\text { preferred prosthesis } \\
\text { at end of trial. } \\
\text { Use of socks } \\
\text { permitted. } \\
-3 \text { months }\end{array}$ & $\begin{array}{l}\text { TSB/pin- } \\
\text { lock versus } \\
\text { PTB/passive } \\
\text {-suction }\end{array}$ & $\begin{array}{l}\text {-Ambulatory } \\
\text { activity } \\
\text { measure to } \\
\text { record number } \\
\text { of steps taken } \\
\text { by the } \\
\text { prosthesis } \\
\text {-Limb volume } \\
\text { changes: } \\
\text { software. } \\
\text {-Subject } \\
\text { satisfaction: } \\
\text { PEQ, BPI and } \\
\text { SCS - } \\
\text { Statistical } \\
\text { Analysis }\end{array}$ & $\begin{array}{l}\text { Step Activity: No } \\
\text { statistical significance } \\
\text { Questionnaires: Results } \\
\text { analysed using two-tailed } \\
\text { Wilcoxon matched pairs, } \\
\text { signed, ranked test. } \\
\text {-No difference in self- } \\
\text { reported scales. } \\
\text { Residual Limb Volume: } \\
\text { No difference. } \\
\text { Subject Preference: } \\
(n=1) \text { returned to original } \\
\text { prosthesis } \\
\text { (n=4) TSB/pin-lock } \\
(n=8) \text { PTB/passive- } \\
\text { suction. } \\
\text { Subjective feedback: exit } \\
\text { interview/ telephone } \\
\text { interviews }\end{array}$ & Yes \\
\hline
\end{tabular}




\begin{tabular}{|c|c|c|c|c|c|c|c|c|c|}
\hline $\begin{array}{l}\text { Sutton et al } \\
(2011)\end{array}$ & 3 & Case report & $\begin{array}{l}\text { Documentatio } \\
\mathrm{n} \text { of effects on } \\
\text { patient's } \\
\text { function after } \\
\text { changing from } \\
\text { PTB/ IWBTC } \\
\text { into } \\
\text { TSB/VASS }\end{array}$ & $\begin{array}{l}40 \text { years old male } \\
\text { trauma resulted in } \\
\text { TT amputation and } \\
\text { tendon and muscle } \\
\text { damage }\end{array}$ & $\begin{array}{l}\text { Interviewed three } \\
\text { times after } \\
\text { intervention (one } \\
\text { week, one month and } \\
\text { one year) }\end{array}$ & $\begin{array}{l}\mathrm{PTB} / \\
\text { IWBTC into } \\
\text { TSB/VASS }\end{array}$ & $\begin{array}{l}\text {-Visual } \\
\text { observation of } \\
\text { gait and } \\
\text { balance } \\
\text {-Self-reported } \\
\text { functional } \\
\text { capabilities } \\
\text { (LC15) } \\
\text {-Activities of } \\
\text { Daily Living } \\
\text { (IADL) index } \\
\text {-Qualitative } \\
\text { interview }\end{array}$ & $\begin{array}{l}\text { Initial fitting: } \\
\text { Benefits: -walked unaided } \\
\text { within } 5 \text { minutes of } \\
\text { donning TSB/VASS } \\
\text {-Stability at knee } \\
\text { Blister formation after one } \\
\text { week due to donning } \\
\text { inconsistencies, healed } \\
\text { within } 2 \text { days. } \\
\text { One year: } \\
\text { - } 24 \text { hours/day limb wear } \\
\text { at work } \\
\text {-increased confidence } \\
\text { (stairs and uneven terrain) } \\
\text {-symmetrical gait } \\
\text {-hair regrowth } \\
\text { Harms: } \\
\text {-still unable to stand } \\
\text { unaided on prosthetic side. } \\
\text {-Limitations: } \\
\text { Lack of baseline for } \\
\text { comparison between } \\
\text { IWBTC and EVTSB to } \\
\text { provide quantitative } \\
\text { analysis. }\end{array}$ & Maybe \\
\hline $\begin{array}{l}\text { Klute et al } \\
\text { (2011) }\end{array}$ & $1+$ & $\begin{array}{l}\text { Randomised } \\
\text { crossover }\end{array}$ & $\begin{array}{l}\text { Investigate } \\
\text { effects of } \\
\text { TSB/VASS } \\
\text { versus } \\
\text { PTB/pin-lock } \\
\text { suspension }\end{array}$ & $\begin{array}{l}\text { 1-unilateral TT, able } \\
\text { to walk for } 30 \text { min, } \\
18-70 \text { years old, } \\
\text { diabetic or } \\
\text { dysvascular, at least } \\
1 \text { year prosthetic use } \\
\text { 2- }(\mathrm{n}=20) /(\mathrm{n}=5) \\
\text { 3- between } 18-70 \\
\text { years old } \\
4 \text { and } 5 \mathrm{n} / \mathrm{a} \\
\text { Withdrew early } \\
(\mathbf{n}=\mathbf{6}) \\
\text { Another }(\mathbf{n}=\mathbf{1 2}) \\
\text { terminated before } \\
\text { completion } \\
\text { Other causes of } \\
\text { termination }(\mathbf{n}=\mathbf{4}) \\
\text { Completed trial } \\
(\mathbf{n}=\mathbf{5})\end{array}$ & $\begin{array}{l}\text { Two limbs } \\
\text { manufactured for } \\
\text { each subject. } \\
\text { TSB/VASS } \\
\text { PTB/pin-lock } \\
\text { Use of socks } \\
\text { permitted as } \\
\text { required. } \\
\text {-3-weeks }\end{array}$ & $\begin{array}{l}\text { TSB/VASS } \\
\text { versus } \\
\text { PTB/pin- } \\
\text { lock }\end{array}$ & $\begin{array}{l}\text {-Activity Level } \\
\text {-Residual } \\
\text { Limb Volume } \\
\text {-Pistoning } \\
\text {-PEQ }\end{array}$ & $\begin{array}{l}\text { Activity level: PTB/pin- } \\
\text { lock, significantly higher } \\
\text { Limb volume: no } \\
\text { statistical significance } \\
\text { Modelling results: pin- } \\
\text { lock residuum will } \\
\text { increase in volume by } \\
4.5 \% \\
\text { TSB/VASS pre-exercise, } \\
\text { volume increase } 4.1 \% \text { and } \\
6.3 \% \text { post-exercise } \\
\text { Pistoning: TSB/VASS } \\
\text { less } \\
\text { Subjects reported for } \\
\text { PTB/pin-lock: } \\
\text {-residuum health better } \\
\text {-easier ambulation } \\
\text {-donning is less frustrating }\end{array}$ & No \\
\hline
\end{tabular}




\begin{tabular}{|c|c|c|c|c|c|c|c|c|c|}
\hline $\begin{array}{l}\text { Traballesi et } \\
\text { al (2012) }\end{array}$ & $1++$ & $\mathrm{RCT}$ & $\begin{array}{l}\text { Investigate } \\
\text { effects of } \\
\text { TSB/VASS } \\
\text { versus } \\
\text { TSB/passive- } \\
\text { suction in TT } \\
\text { amputees with } \\
\text { wounds or } \\
\text { ulcers }\end{array}$ & $\begin{array}{l}\text { 1-Presence of a } \\
\text { wound dehiscence as } \\
\text { a surgical } \\
\text { complication or an } \\
\text { ulcer due to localised } \\
\text { pressure from a } \\
\text { poorly fitting } \\
\text { prosthetic socket - } \\
\text { absence of severe } \\
\text { comorbidities and } \\
\text { phantom pain. } \\
\text { 2- recruited: }(n=20) \\
((n=17) \text { men - }(n=3) \\
\text { women)/ }(n=17) \\
\text { analysed }(n=3) \\
\text { dropped of the CG } \\
\text { 3- } 18-80 \text { years old } \\
\text {-male } 85 \% \text { female } \\
\text { 15\% } \\
\text { 4: N/A }\end{array}$ & $\begin{array}{l}\text { Random assignment } \\
\text { to groups. } \\
\text { TSB/passive-suction } \\
\text { (Control Group CG) } \\
\text { TSB/VASS } \\
\text { (Experimental Group } \\
\text { EG) } \\
\text { Method of } \\
\text { concealment: simple } \\
1: 1 \text { allocation ratio, } \\
\text { subjects selected one } \\
\text { of two sealed } \\
\text { envelopes from a } \\
\text { box. } \\
\end{array}$ & $\begin{array}{l}\text { Both groups } \\
\text { did not use a } \\
\text { liner, no } \\
\text { dressing } \\
\text { applied, } \\
\text { except } \\
\text { night-time } \\
\text { dressing } \\
\text { with } \\
\text { TSB/VASS } \\
\text { group } \\
\text { Same } \\
\text { medication } \\
\text { given to all } \\
\text { participants. }\end{array}$ & $\begin{array}{l}\text { Data collected } \\
8 \text { times and } \\
\text { during follow- } \\
\text { up } 3 \text { times. } \\
\text { Total } \\
\text { observation } \\
\text { period: }(n=36) \\
\text { weeks }\end{array}$ & $\begin{array}{l}\text { Walking capabilities: } \\
\text {-Ambulatory skills: LCI } \\
\text {-Pain: No statistical } \\
\text { significance. } \\
\text {-Wound/ulcer } \\
\text { dimensions: } \\
\text { No statistical significance } \\
\text { for mean wound perimeter } \\
\text { CG subjects at week } 20 \\
\text { did not have full wound } \\
\text { closure. Minor statistical } \\
\text { significance. } \\
\text {-Time of ambulation } \\
\text { since intervention: } \\
\text { CG significantly longer } \\
\text { time } \\
\text {-Statistical analysis: } \\
\text { Baseline: no statistical } \\
\text { significance } \\
\text { Follow-up: } \\
\text { EG subjects used } \\
\text { prosthesis significantly } \\
\text { longer than CG. Improved } \\
\text { at } 4 \text { months, remained } \\
\text { higher with EG }\end{array}$ & No \\
\hline $\begin{array}{l}\text { Brunelli et al } \\
\text { (2013) }\end{array}$ & $1+$ & $\begin{array}{l}\text { Randomised } \\
\text { crossover } \\
\text { study }\end{array}$ & $\begin{array}{l}\text { Compare } \\
\text { effect of } \\
\text { TSB/HIS with } \\
\text { TSB/passive- } \\
\text { suction }\end{array}$ & $\begin{array}{l}\text { 1-unilateral TT } \\
\text { 2-(n=10) } \\
3-(20-65) \text { years old } \\
4-\mathrm{n} / \mathrm{a} \\
5-\mathrm{n} / \mathrm{a}\end{array}$ & $\begin{array}{l}\text { Useful flowchart } \\
\text { provided. } \\
\text { Observational } \\
\text { evaluation: } \\
\text { TSB/suction } \\
\text { outcome measures. } \\
\text { New socket } \\
\text { TSB/HIS } \\
\text { maintaining } \\
\text { remained } \\
\text { componentry. } \\
\text { Evaluation in-term } \\
\text { after } 2 \text { and } 5 \text { weeks } \\
\text { of TSB/HIS use } \\
\\
-7 \text { weeks }\end{array}$ & $\begin{array}{l}\text { TSB/HIS } \\
\text { versus } \\
\text { TSB/passive } \\
\text {-suction }\end{array}$ & $\begin{array}{l}\text {-Pistoning } \\
\text { Test } \\
\text {-Energy Cost } \\
\text { of Walking } \\
\text { (ECW): } \\
\text { prosthesis } \\
\text { efficiency } \\
\text {-PEQ and } \\
\text { HSQ } \\
\text {-Functional } \\
\text { mobility: } \\
\text { Timed } \\
\text { Up\&Go Test } \\
\text { (TUGT) and } \\
\text { the Locomotor } \\
\text { Capability } \\
\text { Index (LCI) } \\
\text {-Time to } \\
\text { achieve } \\
\text { suspension } \\
\text { stability by } \\
\text { HIS }\end{array}$ & $\begin{array}{l}\text { Pistoning/vertical } \\
\text { displacement: } \\
\text { significantly reduced } \\
\text { pistoning with TSB/HIS } \\
\text { ECW: no statistical } \\
\text { significance } \\
\text {-Improved LCI and TUGT } \\
\text { with TSB/HIS Statistical } \\
\text { significance with } \\
\text { TSB/HIS in HSQ. } \\
\text { Limitations: } \\
\text {-Small sample size } \\
\text {-Generalisation not } \\
\text { possible due to specific } \\
\text { activity level and } \\
\text { componentry } \\
\text {-High activity levels may } \\
\text { have affected results } \\
\text { significance. }\end{array}$ & $\begin{array}{l}\text { Yes } \\
\text { (partially } \\
\text { funded by } \\
\text { Ossur and } \\
\text { used Ossur } \\
\text { liners) }\end{array}$ \\
\hline
\end{tabular}




\begin{tabular}{|c|c|c|c|c|c|c|c|c|c|}
\hline $\begin{array}{l}\text { Gholizadeh } \\
\text { et al (2012) }\end{array}$ & $1-$ & $\begin{array}{l}\text { Crossover } \\
\text { non-RCT }\end{array}$ & $\begin{array}{l}\text { Evaluate } \\
\text { pistoning at } \\
\text { prosthetic } \\
\text { socket-liner } \\
\text { interface } \\
\text { during gait } \\
\text { and assess } \\
\text { patients' } \\
\text { satisfaction } \\
\text { with HIS and } \\
\text { pin-lock } \\
\text { liners. }\end{array}$ & $\begin{array}{l}\text { 1-unilateral TT, pain } \\
\text { free, intact skin, } \\
\text { residual limb length } \\
\text { of } \leq 13 \\
2-(n=10) d \\
3-45.8 \text { years } \\
4-n / a \\
5-n / a\end{array}$ & $\begin{array}{l}\text {-Each patient casted } \\
\text { twice for TSB } \\
\text { socket, check socket } \\
\text { used to assess fit } \\
\text { definitive socket } \\
\text { supplied security for } \\
\text { security } \\
\text {-Pistoning evaluation } \\
\text { performed using } \\
\text { Vicon system } \\
\text {-Five trials per } \\
\text { subject recorded on } \\
\text { an } 8 \mathrm{~m} \text { walkway. } \\
\text {-PEQ for satisfaction }\end{array}$ & $\begin{array}{l}\text { TSB/HIS } \\
\text { versus } \\
\text { TSB/pin- } \\
\text { lock }\end{array}$ & $\begin{array}{l}\text { PEQ: } \\
\text { satisfaction } \\
\text { with } \\
\text { suspension } \\
\text { Pistoning } \\
\text { evaluation: } \\
\text { effectiveness of } \\
\text { suspension }\end{array}$ & $\begin{array}{l}\text { Pistoning: } \\
\text { TSB/HIS reduced. } \\
\text {-Motion analysis: } \\
\text { TSB/HIS significantly less } \\
\text { pistoning. } \\
\text { PEQ: TSB/pin-lock } \\
\text { satisfaction higher } \\
\text { Donning/doffing: } \\
\text { TSB/pin-lock higher } \\
\text { Socket fit: TSB/HIS } \\
\text { better. }\end{array}$ & Partially yes \\
\hline $\begin{array}{l}\text { Board et al } \\
(2001)\end{array}$ & 1- & $\begin{array}{l}\text { Crossover } \\
\text { RCT }\end{array}$ & $\begin{array}{l}\text { Compare } \\
\text { between } \\
\text { passive- } \\
\text { suction and } \\
\text { VASS socket } \\
\text { conditions }\end{array}$ & $\begin{array}{l}\text { 1: TT unilateral, } \\
\text { traumatic, amputees, } \\
\text { could walk } 30 \\
\text { minutes on a } \\
\text { treadmill } \\
\text { 2: }(\mathrm{n}=11 \text { ) in } \\
\text { pistoning analysis / } \\
\text { (n=10) volume \& } \\
\text { gait. } \\
\text { No information } \\
\text { provided on the } \\
\text { missing participant. } \\
\text { 3: } 45 \text { years old (32- } \\
\text { 64y) } \\
\text { 4: } \text { N/a } \\
\mathbf{5 :} \text { trauma } \\
\mathbf{6}: \text { mean }=15.2 \text { years }\end{array}$ & $\begin{array}{l}\text { Custom urethane } \\
\text { liners, suspension } \\
\text { sleeves and acrylic } \\
\text { copolymer check } \\
\text { sockets. } \\
\text { Vacuum-casting } \\
\text { utilised. } \\
\text { Liners } 10 \% \text { approx. } \\
\text { undersized and } \\
\text { sockets by } 5 \% \text {. } \\
\text { Outcome measures } \\
\text { were applied } \\
\text { randomly. }\end{array}$ & $\begin{array}{l}\text {-Pistoning: } \\
\text { x-ray } \\
\text {-Volume: } \\
\text { water } \\
\text { displacemen } \\
\text { t before and } \\
\text { after } \\
\text { walking } \\
\text { measured } \\
\text { by single } \\
\text { sample } t \text { - } \\
\text { tests. } \\
\text {-Gait } \\
\text { symmetry: } \\
\text { recorded by } \\
\text { two } 60 \mathrm{~Hz} \\
\text { cameras for } \\
\text { 30min }\end{array}$ & $\begin{array}{l}\text { TSB/passive- } \\
\text { suction (CG) } \\
\text { TSB/VASS } \\
\text { (EG) }\end{array}$ & $\begin{array}{l}\text { Significant difference } \\
\text { amongst outcome } \\
\text { measures recorded in } \\
\text { favour of vacuum } \\
\text { suspension. } \\
\text {-N/a }\end{array}$ & Yes \\
\hline
\end{tabular}




\section{$\underline{\text { Results and discussion: }}$}

Papers obtained were divided into three sections: reviews, socket design clinical studies and studies investigating suspension techniques. Each table illustrates the main findings (PICO: Patient/Population, Intervention, Comparator and Outcomes), grade of evidence ${ }^{30}$ and the presence of commercial bias.

\section{Literature reviews:}

After initial screening, four reviews were deemed suitable against the criteria. A recent and comprehensive systematic review on TT socket design by Safari and Meier (2015), consists of two parts measuring qualitative and quantitative outcomes ${ }^{27,31}$. Gholizadeh et al., (2014), investigated TT suspension systems following a systematic approach ${ }^{32}$. Richardson and Dillon (2017),

reviewed literature investigating user experience of TT liners systematically ${ }^{33}$ while Baars and Geertzen (2005) reviewed literature that investigates the possible advantages of SLs in TT prosthetics $^{34}$.

\section{Socket design reviews: (Table 2)}

Safari and Meier (2015), compared four TT socket designs including PTB/TSB. They listed hydrostatic and VASS as separate designs rather than a different shape capture mechanism in the former and socket suspension method in the latter ${ }^{27}$. Hereafter authors found that the misperception in socket design and suspension prevents drawing a solid conclusion on the suitability of socket design/suspension. Nevertheless, in part one it was concluded that higher activity levels and increased satisfaction were achieved by TSB compared to PTB sockets. Further, ease of donning/doffing was correlated to suspension-mechanisms, which significantly impacted patient satisfaction. Moreover, they found perspiration, odour and skin irritation were related to use of liners, but all reduced over time. Whilst authors found evidence to support TSB sockets, socket satisfaction ratings were considered controversial as cause of amputation, activity level, age and residuum characteristics were found to impact satisfaction and function ${ }^{27}$.

In part two of the review, authors reported that VASS surpassed other suspension techniques. Followed by: TSB/suction socket, TSB/sleeve suspension and TSB/pin-lock respectively. PTB socket with either sleeve or SC suspension was least effective ${ }^{31}$. 
Overall, it may be concluded that TSB/VASS are of utmost benefit to both user and clinician with regards to the reviewers' thesis statement.

\section{Clinical studies on socket design: (Table 3)}

Yigiter et al., (2002), reported that $75 \%$ of participants chose to keep the TSB prosthesis indicating a high level of satisfaction ${ }^{8}$. Also, it was noted that suspension effectiveness and patient balance were improved with the TSB socket. This could be related to different suspension-mechanisms. Yet, the authors also found that TSB sockets require higher accuracy in shape capture and more difficult to manufacture. Furthermore, oedematous and painful residuums were found to be unsuitable for TSB sockets ${ }^{8}$. This is also mentioned in other literature ${ }^{35,36}$ indicating that longer residuums with redundant soft tissue are contraindicated for TSB due to soft tissue bulging during knee flexion ${ }^{35,36}$

TSB/unknown-suspension was reportedly found superior to PTB/unknown-suspension. ${ }^{8}$ Due to the unidentified suspension methods, this finding is ambiguous to the reviewer, therefore comparisons cannot be made. Yet, authors might have been referring to suspension provided by the design of socket, i.e. SC/SCSP.

Conversely, Manucharian (2011), found that the PTB sockets scored higher comfort levels as reported by subjects ${ }^{4}$. The author also found that comfortable socket fit is significantly reliant on individual factors, such as shape capture consistency ${ }^{4}$. This parallels Safari and Meier's (2015) findings.

From the literature it appears that both socket designs may achieve satisfaction under different circumstances when compared to each other.

Selles et al., (2005) ${ }^{37}$ compared PTB/TSB sockets both with pin-lock suspension. Authors reported that the PTB group spent a significantly higher percentage of time standing and ambulating which might be affected by the initial volume fluctuation caused by new weight-distribution. This is correlated to the increased number of visits post-delivery of TSB sockets ${ }^{37}$. However, PEQ scores were similar which indicates that neither the socket design nor suspension affected satisfaction ${ }^{37}$. 
Also, the economical aspect is the only difference between the designs; being significantly higher with $\mathrm{TSB}^{37}$.

Goh et al., (2004) ${ }^{38}$ reported that skill and upper limb dexterity required for pressure cast is minimised, further decreasing casting time and subsequently; cost. Which contraindicates Selles et al.,'s (2005), findings. Yet, one subject in Goh et al.,'s study, reported continuous high pressures proximally in the PTB socket. This is possibly caused by the stretch effect over the soft tissues caused by localised pressure in weight-bearing areas as per socket design biomechanics ${ }^{5,9}$. Though PTB sockets are designed to alleviate pressure from the distal end, another participant experienced distal pressure in the TSB socket due to fibular protrusion. Other participants reported similar pressure intervals during gait with both socket designs ${ }^{38}$.

It is therefore difficult to draw conclusions on optimum prescription of designs as each must be tailored to individual needs of the user.

Goh et al., (2004) $)^{38}$, Manucharian $(2011)^{4}$ and Selles et al., (2005) ${ }^{37}$, all stated that TSB (Handsoff) sockets required less manufacturing time as the requirement for positive cast modification is negated; thus less dexterity is required. Further, production expenses are reduced, -except Selles et al., (2005)- henceforth decreasing error factors for users and facilitating shape capture consistency ${ }^{37,38}$.

Yet, Selles et al., (2002), reported higher visits with TSB sockets after fitting which is a result of the reduction in volume due to a different pressure distribution ${ }^{37}$. Manucharian (2011), also reported that discomfort with $\mathrm{TSB} / \mathrm{HC}$ was attributed to volume fluctuations accompanied with TSB socket due to pressure redistribution ${ }^{4}$ which is similar to Selles et al's (2005) finding ${ }^{4,37,39}$.

\section{Suspension reviews: (Table 4)}

Gholizadeh et al., (2014) $)^{32}$, found that the rate of pistoning within the socket is a good indicator of suspension's quality. Displacement of residuum within the socket was diminished with suction compared to other suspension-mechanisms. However, suction systems were found to increase the difficulty of donning/doffing and are contraindicated in the presence of volume fluctuations. 
Which again repeats the findings of previously mentioned literature ${ }^{32}$. Pressure was found to be distributed more evenly with use of thicker liners however liners resulted in higher perspiration compared to a pe-lite interface ${ }^{32}$.

\section{Liners reviews: (Table 4)}

Richardson and Dillon (2017) $)^{33}$, findings were parallel to Safari and Meier's, $(2015)^{27,31,33}$. Baars and Geertzen $(2005)^{34}$, concluded that limited evidence significantly supports the advantages of SLs. their literature review showed a good indication of suspension improvement when aided by a liner. Furthermore, a positive impact on walking is noted, outdoor walking distances were increased and dependence on walking aids decreased ${ }^{34}$.

Henceforward, a SL/TSB may be preferred if perspiration was not reported as a source of hindrance by the user and if proven to reduce as mentioned in part one of Safari and Meier's, (2015) review.

\section{Clinical studies on suspension: (Table 5)}

Narita et al., (1997), concluded that SL/TSB suspension is superior to PTB's ${ }^{39}$. The x-ray diagrams in the study, display that TSB sockets were suspended by pin-lock. Authors did not disclose the PTB socket suspension-mechanism and referred to it as 'conventional'. In the introduction they discussed that PTB sockets were conventionally suspended by a thigh cuff, it is therefore assumed that experimenters utilised either a thigh cuff or an equivalent to suspend the prosthesis ${ }^{39}$. Moreover, nine subjects were recruited, one of which, was a bilateral amputee hence 10 residuums evaluated. Yet, only three cases were discussed in the assessment of suspension, with no justification provided. Ambiguity in the study methodology limits the reliability of the results.

Sutton et al., (2011), stated that increased stability is achieved in a shorter time with TSB/VASS compared to PTB with weight-bearing thigh cuff. Significant improvement in skin condition was also found. Further, balance improvement, gait symmetry, variant cadence were observed within only a year of TSB/VASS use ${ }^{40}$.

Additionally, Klute et al., $(2011)^{41}$, reported improved socket fit with TSB/VASS with regards to reduced pistoning. Satisfactory fit was achieved in a shorter time and with fewer check sockets for 
PTB/pin suspension ${ }^{41}$. Consequently, this could confirm Selles et al.,'s (2005), finding that TSB sockets could be more costly when more diagnostic sockets are required to optimise fit. Fewer steps were taken when VASS sockets were assessed, if combined with PEQ results, preference in favour of $\mathrm{PTB} / \mathrm{pin}$ suspension is suggested $^{41}$. TSB/VASS maintained constant limb volume after treadmill walk, whereas slight reduction in volume after the walk was scored with PTB/pin-lock ${ }^{41}$. The superior suspension from VASS was thought to be the main factor in the prevention of volume $\operatorname{loss}^{41}$ and improved stability ${ }^{34}$.

Board et al., (2001) ${ }^{23}$, reported improved suspension, gait symmetry, stance duration and step length with TSB/VASS. Pistoning is also reduced with VASS, therefore a better fit is obtained from VASS over suction resulting in reduced skin problems and improved gait symmetry ${ }^{23}$.

Moreover, all significance reached was in favour of TSB/VASS ${ }^{23}$. Optimal socket fit of TSB/VASS would normally maintain volume or minimally increase the volume of the residuum. The gain in volume reported was thought to be attributable to other variables including; participants experiencing gain in water mass or wearing a prosthetic sock for two-hours before donning TSB/VASS ${ }^{23,42,43}$. Contrariwise, the suction group scored loss in volume, suggesting fluid had been drawn out of residuum due to proximal negative pressures ${ }^{23}$.

However, it cannot be said that TSB/VASS would serve the user better with PEQ's ${ }^{41}$ results considered.

Nevertheless, residuum health scored higher with $\mathrm{PTB} /$ pin-lock regarding rash formation, sores or blisters, ingrown hairs, swelling affecting socket fit, sweat and odour ${ }^{41}$. Moreover, approximately double the activity levels were reported with PTB/pin-lock than with TSB/VASS by Klute et al., (2011) which could be related to donning inconsistencies ${ }^{41}$. Improved skin condition was recurrently reported with TSB/VASS. Evidence showed that TSB/VASS not only prevents skin problems but also encourages healing ${ }^{41}$. This statement is supported by Traballesi et al.,'s (2012) finding that TSB/VASS negates the requirement for early ambulation such as PPAM aid before the primary prosthesis ${ }^{42}$. Furthermore, VASS subjects were able to walk within days from protocol initiation. Traballesi et al., (2012), stated that wound healing is achieved with TSB/VASS ${ }^{42}$.

It was also reported that suction can be difficult to maintain, particularly at TT level as bony prominences are more evident than with a TF amputee where soft tissue coverage is more 
manifested. In addition, presence of pain or oedema are other contraindications of the TSB/suction socket $^{14,27,31}$. This parallels Safari and Meier's (2015) statement that the suspension-mechanism has an effect on satisfaction and function of socket ${ }^{27,31}$.

Two studies investigated TSB/HIS, results are similar with regards to reduction in pistoning, improved suspension and difficulty of donning/doffing. Whilst Gholizadeh et al., (2012) ${ }^{19}$ and Brunelli et al., $(2013)^{18}$, agreed on superiority of HIS over pin-lock and suction-suspension, Golizadeh et al., (2012) $)^{19}$, reported that as multiple factors affect patient satisfaction, pistoning is not a determinant of socket satisfaction levels from the user perspective. Brunelli et al., (2013) stated that feeling increased stability within the prosthesis as a result of reduced pistoning could primarily determine user satisfaction ${ }^{18}$. However, the comparator was different in both studies and subjects preferred pin-suspension over TSB/HIS in Gholizadeh et al.,'s (2012) ${ }^{19}$ study. Subjects in Brunelli et al's (2013) ${ }^{18}$ study reported improvement in some aspects with TSB/HIS: cosmesis, rate of ambulation, hours of use and general well-being ${ }^{32}$. Although pistoning was significantly reduced, motor capability of $(\mathrm{ECW})$ figures were not improved. This is probably due to the high activity level of subjects recruited. This could be related to either subject fitness or the insensitivity of outcome measures used. Significant improvement in suspension was reported in both systems after seven-weeks, henceforth, authors speculate that it requires seven-weeks to acclimate to the HIS system. Yet, no other studies confirmed this statement ${ }^{18}$.

Coleman et al.,'s (2004), concluded that participants significantly preferred PTB/passive-suction for ambulatory activities over TSB/pin-lock ${ }^{20}$. PEQ results revealed equal satisfaction levels and intensity of ambulation for both systems. This is related to higher comfort consistency over long periods of time with PTB/passive-suction. TSB/pin-lock, scored well for socket comfort over short periods of time but this decreased over longer periods. Reduction in comfort over time could be attributable to increased perspiration and the 'milking phenomenon'. Furthermore, skin irritation proximally and elsewhere was evident. In addition, elastometric liner durability and economical aspects were of concern while neoprene liner durability was questioned, yet affordable. This could indicate that satisfaction is more reliant on the suspension-mechanism in relation to donning/doffing rather than socket design, echoing the findings of Klute et al., (2011). 


\section{Tables 2 and 4:}

Perspiration increased with TSB as reported however, this did not affect patient satisfaction. Yet, ease of donning/doffing was directly correlated with patient satisfaction ${ }^{(24,30)}$. These findings concur with Baars and Geertzen (2005) ${ }^{34}$. However, Safari and Meier (2015) ${ }^{27,31}$, reported that skin problems are reduced with TSB/SL compared to PTB due to even pressure distribution. Nevertheless, skin problems were not resolved by utilising a SL but may be aggravated by the build-up of high levels of perspiration ${ }^{34}$. This is related to sweat build-up due to the confined environment a SL creates.

As concluded from the studies examined, weight-bearing is achieved with TSB more evenly in combination with suction suspension. Amongst suction mechanisms examined, VASS has proven superior to other types with regards to equal weight-bearing between limbs. This parallel's Safari and Meier et al.,'s (2015) findings ${ }^{27,31}$.

\section{Tables 4 and 5:}

Gholizadeh et al., $(2012)^{19}$, reported that TSB achieve increased weight bearing through the prosthesis compared to PTB, improving balance. Authors attributed this to total contact which further improves proprioceptive feedback and pressure distribution ${ }^{19}$. Similar results were found by Sutton et al., (2011), demonstrating that more equal weight distribution is likely to be attributable to the change in socket type ${ }^{40}$.

Difficulty of donning/doffing increased with suction ${ }^{40}$ and improper donning can result in either loss of suction -and failure of the system- or blistering as reported by Sutton et al., (2011) ${ }^{40}$ and Klute et al., (2011) ${ }^{41}$. Pistoning with TSB/VASS was significantly reduced versus PTB/pin. Though authors' stated that TSB/VASS may be easier to don as there is no pin to align, subjects reported lower frustration levels with $\mathrm{PTB} /$ pin. A more optimal fit and reduced pistoning was obtained with TSB/VASS, yet, subjects' favoured PTB/pin over VASS which was related to lower frustration levels with donning. This supports Ghozelideh et al.,'s (2014) Safari and Meier's (2015) and Baars and Geertzen's (2005) statement that patient satisfaction is not affected by the rate of pistoning. 


\section{Tables 3 and 5:}

Improved skin health reported with TSB/VASS, around the fibula head, mid-patella tendon and proximal brim, by Sutton et al..(2011). However, these areas were identified as problematic by one subject in Goh et al., 's (2004) study. Therefore, due to the contradictory findings, it is difficult to draw a comparison about the effect of TSB on skin health.

Board et al., (2001) $)^{23}$, also reported that volume loss was prevented in TSB/VASS. These diurnal fluctuations are important as they contribute to ill-fitting sockets and consequent loss of suspension resulting in skin problems, gait deviations and system failure ${ }^{23}$. If pistoning is minimised, skin breakdown is reduced or expectantly eliminated ${ }^{7,8}$.

TSB/VASS is superior to PTB/pin in terms of socket fit due to reduced pistoning hence improved suspension $^{41}$. Yet subjects favoured PTB/pin. Though, Coleman et al., (2004) ${ }^{20}$ compared $\mathrm{PTB} /$ passive suction to TSB/pin-lock and found that 10 out of 13 subjects chose PTB/passivesuction socket when asked to choose a sole prosthesis. This was thought to be due to inconvenient donning/doffing of TSB/pin-lock, perspiration of gel liner and discomfort due to pin ${ }^{(12)}$.

\section{Tables 3, 4 and 5:}

Superior suspension of TSB/pin-lock sockets are also reported by Narita et al., (1997) and Yigiter et al., (2002), over TSB/unknown-suspension ${ }^{8,39}$. In TSB, the difficulty of maintaining a good fit/suspension is related to the requirement for adequate pressure levels which can subsequently result in volume reduction. This may compromise the socket fit which is proven problematic to the skin and may lead to ulceration ${ }^{39}$. Board et al., (2001) found that TSB/passive-suction contribute to initial loss in volume, due to the new weight-distribution ${ }^{23}$. This was supported by Manucharian $(2011)^{4}$, who found that TSB/passive-suction might have caused volume fluctuations resulting in reduced satisfaction and comfort levels ${ }^{23}$.

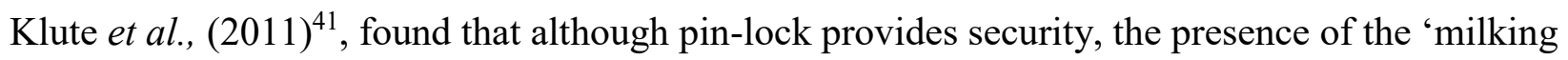
phenomenon' is challenging. Yet, subjects favoured pin-suspension over VASS. This is regarded to lower frustration levels with donning, though pistoning is reduced with VASS thereafter, supporting Ghozelideh et al., 's (2014) and Safari and Meier's (2015) statement that patient satisfaction is not affected by the reduction of pistoning ${ }^{27}$. Furthermore, skin irritation at the proximal end and elsewhere is probably due to 'milking phenomenon' that was reported with 
TSB/pin-lock ${ }^{(12)}$. Finally, with TSB/HIS, Gholizadeh et al., $(2012)^{19}$ reported that subjects stated that the HIS felt like a part of their body due to the firm attachment of the liner with the socket wall. HIS resulted in significantly less pistoning, resolving the 'milking phenomena'. Yet they were more satisfied with the pin-lock ${ }^{31}$. This emphasises on Gholizadeh et al., 's (2014) survey finding that the majority of users prefer TSB/pin-lock. Again reinforcing the fact that pistoning is not a determinant of satisfaction ${ }^{32}$.

Safari and Meier (2015), found that donning/doffing of sockets is pertinent to the suspensionmechanism and has a significant impact on patient satisfaction. Richardson and Dillon (2017), hypothesised that frustration levels decrease when users have previous experience with the design/suspension ${ }^{32}$. Other findings are parallel to Safari and Meier's (2015) in terms of user satisfaction with design/suspension-mechanism with donning/doffing, pistoning and

perspiration ${ }^{27,31}$. Further, Gholizadeh et al., (2014) ${ }^{32}$ reported that comfort is increased with thicker liners as more equal distribution of pressure is achieved. Yet, skin problems were often reported plus the difficulty of donning/doffing ${ }^{31}$.

Literature included was from 12 different countries and included different states/regions from within. This may have affected the consistency of the results obtained due to varying fabrication methods conducted in various regions.

\section{Methodological errors:}

Baars and Geertzen (2005), reported heterogeneity in aeitology, participant selection criteria, age, acclimatisation (table 5) of prosthesis use ${ }^{34}$. A decade later, Safari and Meier (2015), reported very similar limitations ${ }^{27,31}$ indicating that development of research methods in prosthetics is very slow. Similar limitations were found in included studies limiting comparisons made due to the aforementioned vast heterogeneity in design, intervention and comparators.

Yigiter et al., (2002) $)^{8}$, compared TSB with PTB, however, suspension and shape capture mechanisms were not mentioned. The term 'soft liner' was stated as an interface material for both sockets. Moreover, TSB was referred to as 'total contact' obviating the fact that PTB sockets are also total contact. Advantageous results reported in favour of TSB were attributed to 'total contact'. Outcomes that favoured TSB included higher weight-bearing acceptance, improved 
balance and ambulation activities. All of which were related to total contact and good pressure distribution thus increased proprioception and control of the prosthesis ${ }^{13,34}$.

Selles et al., (2005) $)^{37}$ reported that standard deviation discrepancies were high within both groups indicating that some subjects extremely favoured the new prosthesis or older prosthesis, which could be attributable to familiarity or raised expectations of a new socket ${ }^{13,14,27,31}$. Still, only one participant did not keep the new prosthesis at the end of trial (PTB or TSB), according to the authors, indicating that most subjects contradicted themselves. Reviewer anticipates that PEQ was not well explained or due to insensitivity of the PEQ or due to acclimation periods.

Manucharian (2011), reported a significant decrease in comfort correlated to the increase in the number of adjustments made to the HCTSB sockets ${ }^{4}$. This could be related to the experimenter's choice of interface (Pe-lite) to minimise variables. Reviewers indicate that the accuracy and legitimacy of the results could have been affected, as SLs were considered a prime discriminant between the two socket designs due to the material characteristics detailed previously. Though most patients did change the design of socket from their original prosthesis, a significant discrepancy was noted in comfort scores between the changed and non-changed groups in favour of the changed group. Comfort and satisfaction were negatively affected by limb volume fluctuations in the HCTSB socket. Fluctuations could have occurred as a result of gapping within the socket or an undersized socket, with volume increasing to accommodate the additional space 4 .

Details on whether the terminated subjects from Klute et al's (2011), study were included in the analysis or not, are not mentioned ${ }^{41}$.

\section{Conclusion:}

Despite the methodological errors noted in the studies and the commercial bias that may have impacted the accuracy of results, findings of this review are partially consistent with Safari and Meier's ${ }^{27,31}$ review with regards to suspension superiority. Effectiveness of socket design was not clear due to the significant heterogeneity as mentioned by previous authors, therefore, comparisons cannot be effectively made and in some cases, the results are unreliable. However, biomechanically, TSB sockets allow for a more even weight-distribution when combined with 
suction, particularly VASS. Additionally, minor yet promising, evidence is provided on TSB/VASS regarding wound healing and early ambulation. However, PTB sockets are still successfully used and in some studies preferred over TSB. A conclusion on whether preference is due to suspension-mechanism or design itself cannot be drawn. Therefore, systematic reviews must be conducted to normalise acclimation periods for socket design and suspension along with crossover RCTs with larger sample sizes for effective clinical basis to be made for improved clinical practice, with minimal commercial bias.

Word count: 4,965

\section{Acknowledgment:}

The authors would like to thank Mr. Stephanos Solomindis for the time he devoted and his appreciated input and advice. 


\section{$\underline{\text { References: }}$}

1. Scott H, Patel R, Hebenton J. A Survey of the Lower

Limb Amputee

Population in Scotland,

2014

Full Report. In: Scottish Physiotherapy Amputee Research Group; 2017.

2. Klasson B. Appreciation of prosthetic socket fitting from basic engineering principles.

Glasgow (GB): Glasgow GB : National Centre for Training and Education in Prosthetics and Orthotics; 1995.

3. Laing S, Lee PVS, Goh JCH. Engineering a Trans-Tibial Prosthetic Socket for the Lower Limb Amputee. Annals Academy of Medicine Singapore. 2011;40(5):252-259.

4. Manucharian S. An Investigation of Anticipated Comfort Level Trend Differences Between the Hands-on Patellar-Tendon Bearing (PTB) and Hands-off Hydrocast Transtibial Prosthetic Sockets [Master's Thesis]2009.

5. Weber D. Clinical aspects of lower extremity prosthetics : trans tibial, symes and partial foot amputations. Oakville, Ont.: Oakville, Ont. : Elgan Enterprises; 1988.

6. Fergason J, Smith DG. Socket considerations for the patient with a transtibial amputation. Clinical orthopaedics and related research. 1999(361):76-84.

7. Zhang M, Turner-Smith AR, Roberts VC, Tanner A. Frictional action at lower limb/prosthetic socket interface. Medical Engineering \& Physics. 1996;18(3):207-214. 
8. Yiğiter K, Sener G, Bayar K. Comparison of the effects of patellar tendon bearing and total surface bearing sockets on prosthetic fitting and rehabilitation. Prosthetics and orthotics international. 2002;26(3):206-212.

9. Sanders JE, Zachariah SG, Jacobsen AK, Fergason JR. Changes in interface pressures and shear stresses over time on trans-tibial amputee subjects ambulating with prosthetic limbs: comparison of diurnal and six-month differences. Journal of Biomechanics. 2005;38(8):1566-1573.

10. Bakalim G. Experiences with the total-contact prosthesis. Artif Limbs. 1967;11(1):51-57.

11. Safari MR, Rowe P, McFadyen A, Buis A. Hands-Off and Hands-On Casting Consistency of Amputee below Knee Sockets Using Magnetic Resonance Imaging. Scientific World Journal. 2013.

12. Topper AK, Fernie GR. Computer-aided design and computer-aided manufacturing (CAD/CAM) in prosthetics. Clin Orthop Relat Res. 1990(256):39-43.

13. Kapp S. Suspension systems for prostheses. Clin Orthop Relat Res. 1999(361):55-62.

14. Roberts RA. Suction socket suspension for below-knee amputees. Arch Phys Med Rehabil. 1986;67(3):196-199.

15. Kristinsson O. The ICEROSS concept: a discussion of a philosophy. Prosthet Orthot Int. 1993;17(1):49-55.

16. Levy SW. Skin problems of the leg amputee. Prosthet Orthot Int. 1980;4(1):37-44.

17. Wells G. Carl Caspers, CPO: Innovation Sparked by Personal Experience. In: The O\&P Edge; 2013:184-189. 
18. Brunelli S, Delussu AS, Paradisi F, Pellegrini R, Traballesi M. A comparison between the suction suspension system and the hypobaric Iceross Seal-In(R) X5 in transtibial amputees. Prosthet Orthot Int. 2013;37(6):436-444.

19. Gholizadeh H, Abu Osman NA, Eshraghi A, et al. Transtibial prosthetic suspension: less pistoning versus easy donning and doffing. J Rehabil Res Dev. 2012;49(9):1321-1330.

20. Coleman KL, Boone DA, Laing LS, Mathews DE, Smith DG. Quantification of prosthetic outcomes: elastomeric gel liner with locking pin suspension versus polyethylene foam liner with neoprene sleeve suspension. $J$ Rehabil Res Dev. 2004;41(4):591-602.

21. Gholizadeh H, Abu Osman NA, Kamyab M, Eshraghi A, Lúvíksdóttir AG, Wan Abas WAB. Clinical evaluation of two prosthetic suspension systems in a bilateral transtibial amputee. American journal of physical medicine \& rehabilitation. 2012;91(10):894-898.

22. Ferraro C. Outcomes Study of Transtibial Amputees Using Elevated Vacuum Suspension in Comparison With Pin Suspension. Journal of Prosthetics and Orthotics. $2011 ; 23(2): 78-81$.

23. Board WJ, Street GM, Caspers C. A comparison of trans-tibial amputee suction and vacuum socket conditions. Prosthetics and Orthotics International. 2001;25(3):202-209.

24. Foort J. The patellar-tendon-bearing prosthesis for below-knee amputees, a review of technique and criteria. Artificial limbs. 1965;9(1):4-13.

25. Abu Osman NA, Spence WD, Solomonidis SE, Paul JP, Weir AM. The patellar tendon bar! Is it a necessary feature? Medical Engineering \& Physics. 2010;32(7):760-765. 
26. Hall MJ, Shurr DG, Vanbeek MJ, Zimmerman MB. The prevalence of dermatological problems for transtibial amputees using a roll-on liner. Journal of Prosthetics and Orthotics. 2008;20(4):134-139.

27. Safari MR, Meier MR. Systematic review of effects of current transtibial prosthetic socket designs-Part 1: Qualitative outcomes. Journal of Rehabilitation Research and Development. 2015;52(5):491-508.

28. Conference on Priorities in Prosthetic and Orthotic Practice and Murdoch G. Prosthetic and orthotic practice based on a conference held in Dundee, June, 1969. 1970.

29. Day JD. Effectiveness of a Modified Icex ${ }^{\circledR}$ Casting Technique Based on Circumferential Change in Residual Limb Volume. JPO: Journal of Prosthetics and Orthotics. $2013 ; 25(4)$.

30. Healthcare Improvement Scotland. Scottish Intercollegiate Guidelines Network (SIGN). In:2004.

31. Safari MR, Meier MR. Systematic review of effects of current transtibial prosthetic socket designs-Part 2: Quantitative outcomes. Journal of Rehabilitation Research and Development. 2015;52(5):509-526.

32. Gholizadeh H, Abu Osman NA, Eshraghi A, Ali S, Razak NA. Transtibial prosthesis suspension systems: systematic review of literature. Clin Biomech (Bristol, Avon). 2014;29(1):87-97.

33. Richardson A, Dillon MP. User experience of transtibial prosthetic liners: A systematic review. Prosthet Orthot Int. 2017;41(1):6-18.

34. Baars EC, Geertzen JH. Literature review of the possible advantages of silicon liner socket use in trans-tibial prostheses. Prosthet Orthot Int. 2005;29(1):27-37. 
35. Hachisuka K, Nakamura T, Ohmine S, Shitama H, Shinkoda K. Hygiene problems of residual limb and silicone liners in transtibial amputees wearing the total surface bearing socket. Archives of Physical Medicine and Rehabilitation. 2001;82(9):1286-1290.

36. Hachisuka K, Dozono K, Ogata H, Ohmine S, Shitama H, Shinkoda K. Total surface bearing below-knee prosthesis: Advantages, disadvantages, and clinical implications. Archives of Physical Medicine and Rehabilitation. 1998;79(7):783-789.

37. Selles RW, Janssens PJ, Jongenengel CD, Bussmann JB. A randomized controlled trial comparing functional outcome and cost efficiency of a total surface-bearing socket versus a conventional patellar tendon-bearing socket in transtibial amputees. Archives of Physical Medicine and Rehabilitation. 2005;86(1):154-161.

38. Goh JCH, Lee PVS, Chong SY. Comparative study between patellar-tendon-bearing and pressure cast prosthetic sockets. Journal of Rehabilitation Research and Development. 2004;41(3B):491-501.

39. Narita H, Yokogushi K, Shii S, Kakizawa M, Nosaka T. Suspension effect and dynamic evaluation of the total surface bearing (TSB) trans-tibial prosthesis: a comparison with the patellar tendon bearing (PTB) trans-tibial prosthesis. Prosthet Orthot Int. 1997;21(3):175-178.

40. Sutton E, Hoskins R, Fosnight T. Using Elevated Vacuum to Improve Functional Outcomes: A Case Report. JPO: Journal of Prosthetics and Orthotics. 2011;23(4).

41. Klute GK, Berge JS, Biggs W, Pongnumkul S, Popovic Z, Curless B. Vacuum-Assisted Socket Suspension Compared With Pin Suspension for Lower Extremity Amputees: Effect on Fit, Activity, and Limb Volume. Archives of Physical Medicine and Rehabilitation. 2011;92(10):1570-1575. 
42. Traballesi M, Delussu AS, Fusco A, et al. Residual limb wounds or ulcers heal in transtibial amputees using an active suction socket system. A randomized controlled study. Eur J Phys Rehabil Med. 2012;48(4):613-623. 